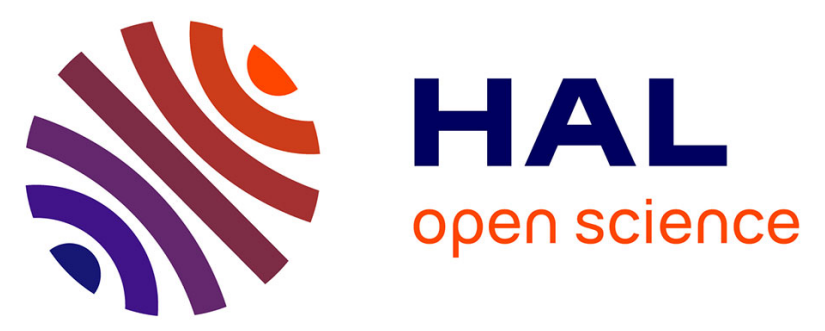

\title{
Application de la technique des oscillations d'intensité de diffraction électronique en incidence rasante à la croissance des semi-conducteurs III-V par épitaxie par jets moléculaires
}

F. Turco, J. Massies, J.P. Contour

\section{To cite this version:}

F. Turco, J. Massies, J.P. Contour. Application de la technique des oscillations d'intensité de diffraction électronique en incidence rasante à la croissance des semi-conducteurs III-V par épitaxie par jets moléculaires. Revue de Physique Appliquée, 1987, 22 (8), pp.827-836. 10.1051/rphysap:01987002208082700 . jpa-00245616

\author{
HAL Id: jpa-00245616 \\ https://hal.science/jpa-00245616
}

Submitted on 1 Jan 1987

HAL is a multi-disciplinary open access archive for the deposit and dissemination of scientific research documents, whether they are published or not. The documents may come from teaching and research institutions in France or abroad, or from public or private research centers.
L'archive ouverte pluridisciplinaire $\mathbf{H A L}$, est destinée au dépôt et à la diffusion de documents scientifiques de niveau recherche, publiés ou non, émanant des établissements d'enseignement et de recherche français ou étrangers, des laboratoires publics ou privés. 


\title{
Application de la technique des oscillations d'intensité de diffraction électronique en incidence rasante à la croissance des semi-conducteurs III-V par épitaxie par jets moléculaires
}

\author{
F. Turco, J. Massies et J. P. Contour \\ Laboratoire de Physique du Solide et Energie Solaire, CNRS - Sophia Antipolis, 06560 Valbonne, France
}

(Reçu le 23 janvier 1987, révisé le 17 mars 1987, accepté le 23 mars 1987)

\begin{abstract}
Résumé. - L'enregistrement des oscillations d'intensité de diffraction des électrons en incidence rasante (RHEED) en cours de croissance épitaxiale par jets moléculaires (EJM), permet la détermination en temps réel de certains paramètres essentiels de la croissance tels que vitesse, composition d'alliage, intensité des flux d'éléments III incidents à la surface et coefficients de collage des éléments III. Nous donnons des exemples de l'utilisation de cette technique obtenus dans le cas de la croissance de $\mathrm{GaAs}, \mathrm{AlAs}, \mathrm{Al}(x) \mathrm{Ga}(1-x) \mathrm{As}$ et $\mathrm{Al}(1-x) \operatorname{In}(x)$ As sur substrats GaAs. Nous discutons des états de rugosité de l'interface de croissance observés pour ces différents matériaux et de leurs évolutions en fonction des paramètres de croissance. D'autre part, nous reportons l'observation pour la première fois du phénomène des oscillations de RHEED pendant la croissance $\mathrm{Ga}(1-x) \operatorname{In}(x)$ As et $\mathrm{Al}(1-x) \operatorname{In}(x)$ As en accord de maille sur substrat InP.
\end{abstract}

\begin{abstract}
Reflection high energy electron diffraction (RHEED) oscillations recorded during molecular beam epitaxy (MBE) allows the determination in real time of some of the main growth parameters of III-V semiconductors such as growth rate, element III fluxes impinging at the surface and sticking coefficients of the elements III. Illustrations of this technique are given for $\mathrm{GaAs}, \mathrm{AlAs}, \mathrm{Al}(x) \mathrm{Ga}(1-x) \mathrm{As}$ and $\mathrm{Al}(1-x) \operatorname{In}(x)$ As epitaxial growth onto GaAs substrates. The growth interface roughness for these different materials is investigated versus growth parameters. We also report for the first time the observation of RHEED oscillations phenomenon during the growth of $\mathrm{Ga}(1-x) \operatorname{In}(x)$ As and $\mathrm{Al}(1-x) \operatorname{In}(x)$ As lattice matched on InP substrates.
\end{abstract}

\section{Introduction.}

L'enregistrement des oscillations d'intensité de diffraction des électrons en incidence rasante (RHEED) au cours de la croissance épitaxiale par jets moléculaires (EJM) permet la détermination en temps réel de certains paramètres essentiels de la croissance. Après avoir brièvement rappelé le modèle d'interprétation des oscillations de RHEED proposé par Neave et al. [1], nous présentons des exemples de la détermination directe des paramètres de croissance suivants :

- vitesse de croissance et composition d'alliage,

- intensité des flux moléculaires d'éléments III incidents à la surface,

- coefficient de collage des éléments III.

Nous reportons ensuite les résultats obtenus par cette technique sur l'influence de certains paramètres de croissance, tels que flux d'élément $V$ et tempéra- ture du substrat, sur la rugosité à l'interface de croissance dans le système ( $\mathrm{Al}, \mathrm{Ga}) \mathrm{As} / \mathrm{GaAs}$.

Enfin, nous avons également observé le phénomène des oscillations de RHEED dans le système (AlGa)InAs en accord paramétrique sur InP ou contraint sur GaAs et quelques résultats préliminaires concernant ce système sont présentés.

\section{Détails expérimentaux.}

Les oscillations de RHEED ont été enregistrées sur un système Riber 2300 équipé d'un canon à électrons 0-10 keV. Les électrons arrivent sous incidence rasante sur la surface de l'échantillon en cours de croissance. Le faisceau émergent comprend une partie réfléchie et une partie diffractée qui donnent respectivement sur l'écran fluorescent une tache spéculaire et un ensemble de raies d'ordre entier ou fractionnaire. Pour l'ensemble des résultats reportés 
ici seule la variation de l'intensité lumineuse de la tache spéculaire, moins sensible aux effets dynamiques de diffraction, est considérée. Cependant, certains détails des oscillations de la tache spéculaire restent fonction de l'angle d'incidence des électrons à la surface, à cause de la part de son intensité résultant d'effets de diffraction multiple [2,3]. C'est en particulier le cas du transitoire observé au départ de la croissance. Afin de permettre une étude comparative des oscillations enregistrées, l'angle d'incidence des électrons a été maintenu constant et égal à $0,5^{\circ}$. L'intensité lumineuse de la tache spéculaire est collimatée au moyen d'un objectif de microscope à l'entrée d'une fibre optique, reliée à un photomultiplicateur lui-même connecté à un enregistreur (Fig. 1). Les raies de diffraction permettent quant à elles de déterminer la symétrie de la reconstruction de surface.

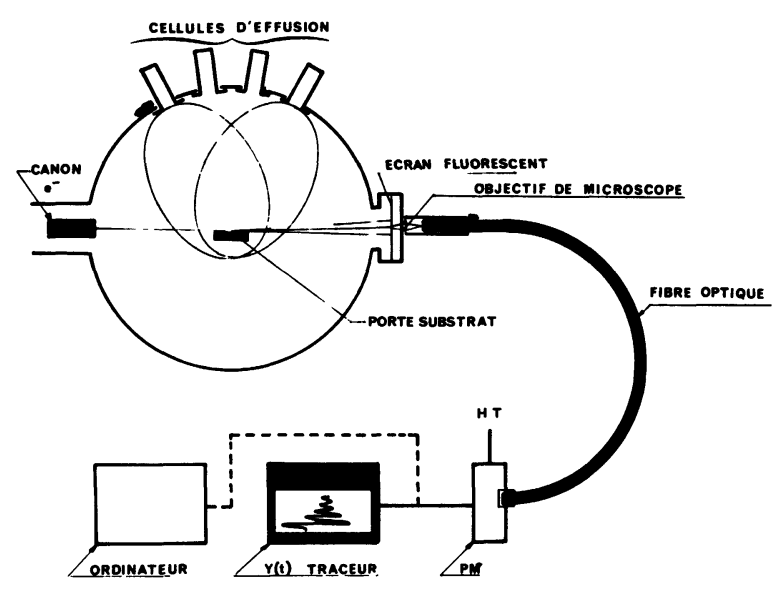

Fig. 1. - Schéma du dispositif d'enregistrement des oscillations de RHEED.

[Schematic illustration of the RHEED oscillations set-up.]

\section{Interprétation des oscillations.}

Le modèle d'interprétation des oscillations de RHEED proposé par Neave et al. [1] est ici appliqué aux variations d'intensité de la tache spéculaire qui peuvent être expliquées en terme de variations de réflectivité de l'interface de croissance dues à l'évolution de la rugosité liée aux mécanismes de croissance. Lors d'un mode de croissance couche par couche, type Franck van der Merwe, la croissance procède par nucléation 2D. L'évolution de la rugosité est alors corrélée à la variation séquentielle de la densité de nucléites 2D au cours de la croissance, de l'initiation à l'achèvement de chaque couche de matériau considéré. Dans ce modèle idéal, la densité de nucléites $2 \mathrm{D}$ sera maximale pour un recouvrement équivalent à la demi-couche. Ceci correspondra au maximum de rugosité et donc au minimum de réflectivité de la surface. Au-delà, la taille des îlots augmente et la réflectivité s'accroît jusqu'à un maximum relatif à la formation d'une couche complète. Ce modèle schématique explique le caractère oscillatoire de l'intensité réfléchie en cours de croissance. Si la croissance n'est pas idéalement $2 \mathrm{D}$, la croissance de la $(n+1)$-ième couche pourra débuter avant que la $n$-ième ne soit complète. Cette perturbation du modèle idéal a été proposée par Neave et al. [4] pour expliquer l'amortissement général des oscillations avec le temps de croissance (Fig. 2). Lors d'un arrêt de croissance avec maintien de la température du substrat et du flux d'arsenic, la surface se lisse par migration d'atomes et retrouve sa réflectivité initiale (Fig. 2).

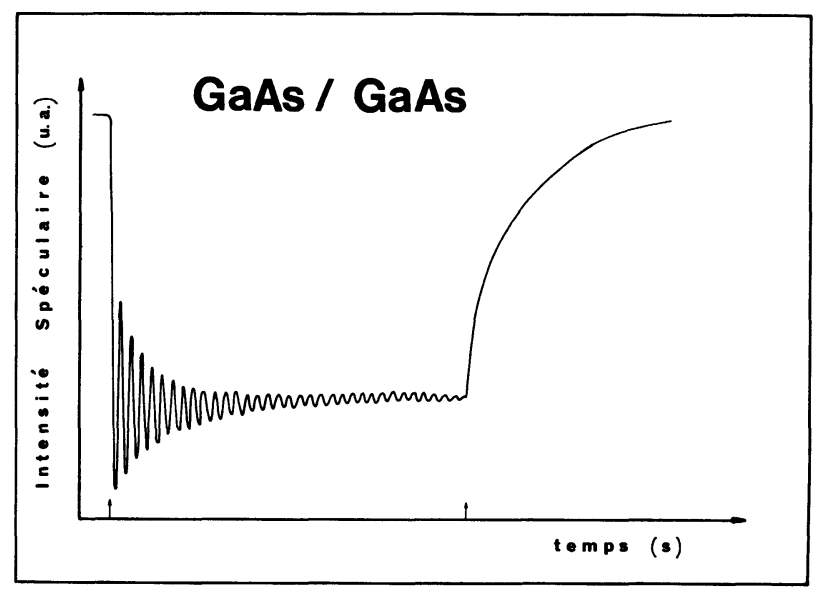

Fig. 2. - Oscillations de RHEED (tache spéculaire, azimut [110]) pendant la croissance de $\mathrm{GaAs} / \mathrm{GaAs}$ à $600^{\circ} \mathrm{C}$ avec récupération de l'intensité initiale à l'arrêt de croissance (les flèches portées en abscisse correspondent au début et à l'arrêt de croissance).

[RHEED oscillations (specular beam intensity, [110] azimuth) during $\mathrm{GaAs} / \mathrm{GaAs}$ growth at $600{ }^{\circ} \mathrm{C}$ and the recovering of the initial intensity at the end of growth (arrows in abscissa correspond to the beginning and the end of the growth).]

\section{Informations directes sur les paramètres de crois- sance.}

4.1 VITESSE DE CROISSANCE ET COMPOSITION D'Alliage. - La période $T$ des oscillations (exprimée en s) correspond au temps de croissance d'une monocouche moléculaire III-V (MC), c'est-à-dire, pour la croissance selon [001], à une épaisseur de matériau de $a / 2$ où $a$ est le paramètre de maille du semi-conducteur considéré exprimé en $\AA$. On obtient directement la vitesse de croissance en $\AA / \mathrm{s}$ par $v(\mathrm{III}-\mathrm{V})=(1 / T) \times(a / 2)$ ou en $\mathrm{MC} / \mathrm{s} \quad \mathrm{par}$ $v(\mathrm{III}-\mathrm{V})=1 / T$.

En ce qui concerne par exemple la croissance de $\mathrm{Al}(x) \mathrm{Ga}(1-x)$ As, la détermination de la composition d'alliage est directement obtenue à partir des 


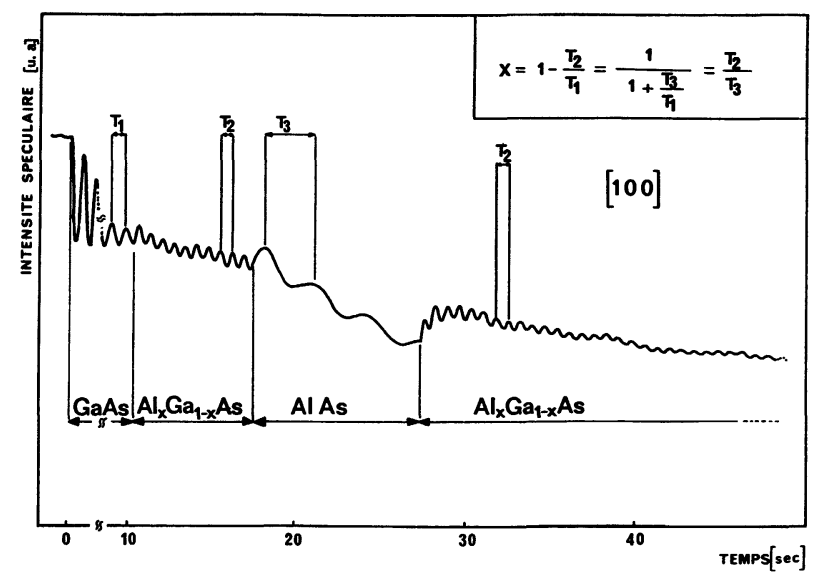

Fig. 3. - Oscillations de RHEED pendant la croissance de $\mathrm{GaAs} / \mathrm{GaAs}, \mathrm{Al}(x) \mathrm{Ga}(1-x)$ As $/ \mathrm{GaAs}$ et AlAs/ $\mathrm{GaAs}$ et détermination de la composition en aluminium de l'alliage $\mathrm{Al}(x) \mathrm{Ga}(1-x) \mathrm{As}$, dans le cas où le coefficient de collage de $\mathrm{Ga}$ est égal à 1 (sinon seule l'expression $x=T_{2} / T_{3}$ est utilisée).

[RHEED oscillations during GaAs/GaAs, $\mathrm{Al}(x) \mathrm{Ga}(1-x) \mathrm{As} / \mathrm{GaAs}$ and AlAs/GaAs growths and determination of the $\mathrm{Al}(x) \mathrm{Ga}(1-x)$ As alloy aluminium concentration, when the Ga sticking coefficient is equal to unity (if not only $x=T_{2} / T_{3}$ is used).]

vitesses de croissance de deux des composés suivants : $\mathrm{GaAs}, \mathrm{Al}(\boldsymbol{x}) \mathrm{Ga}(1-x) \mathrm{As}$ et AlAs (Fig. 3). Soient $T_{1}, T_{2}$ et $T_{3}$ les périodes des oscillations des croissances respectives des trois composés GaAs, $\mathrm{Al}(x) \mathrm{Ga}(1-x)$ As et AlAs, alors :

$$
x=1-\left(T_{2} / T_{1}\right)=T_{2} / T_{3}=T_{1} /\left(T_{1}+T_{3}\right) .
$$

Cette équation n'est en fait strictement valable que dans la zone de température de croissance $T_{\mathrm{s}}$ où le coefficient de collage Ga est égal à 1. Dans les conditions normales de vitesse de croissance $(\sim 1 \mathrm{MC} / \mathrm{s}$ ) et de flux d'arsenic (pression dans le flux $\sim 1 \times 10^{-5}$ torr), cette zone correspond à $T_{\mathrm{s}}<650^{\circ} \mathrm{C}$. Au-delà de cette température, le coefficient de collage de $\mathrm{Ga}$ diminue, mais sa décroissance est limitée, comme nous allons le voir, par la présence d'aluminium. La détermination précise de $x$ ne peut alors être obtenue que par la relation $x=T_{2} / T_{3}$.

\subsection{ETALONNAGE DES FLUX D’ÉLÉMENTS} III. - La vitesse de croissance à température de substrat et pression d'arsenic constantes est fonction directe des températures des cellules d'éléments III. Les flux incorporés exprimés en at $/\left(\mathrm{s} . \mathrm{cm}^{2}\right.$ ) (égaux aux flux émis dans la limite des coefficients de collage égaux à 1) peuvent être facilement et rapidement étalonnés en fonction de la température $T_{\text {c }}$ de la cellule par mesure de la variation de la période $T$ des oscillations de RHEED (Fig. 4) :

$$
\phi(\text { III })=2 /\left(T \cdot a^{2}\right) \quad \text { avec } T \text { en s et } a \text { en cm }
$$

où

$$
\phi(\text { III })=A \cdot \exp \left(-E(\text { III }) / k T_{\mathrm{c}}\right) .
$$

Ceci permet, dans le cas de coefficients de collage égaux à 1 , de déterminer les énergies d'activation pour l'évaporation du gallium et de l'aluminium $E(\mathrm{Ga})=2,6 \mathrm{eV}$ et $E(\mathrm{Al})=3,0 \mathrm{eV}$ (Fig. 4).

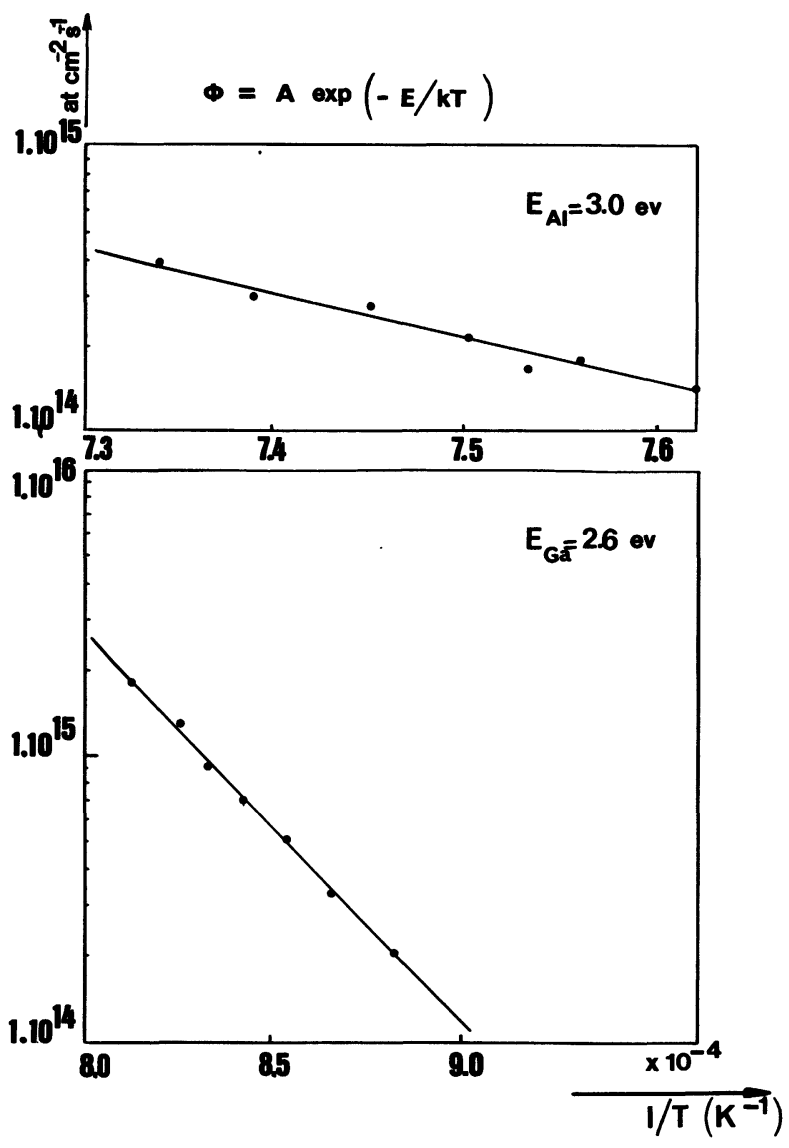

Fig. 4. - Intensité des flux d'éléments III ( $\mathrm{Al}$ et $\mathrm{Ga}$ ) en fonction de l'inverse de la température des cellules correspondantes.

[Elements III ( $\mathrm{Al}$ and $\mathrm{Ga}$ ) fluxes intensity versus corresponding cells reciprocal temperature.]

4.3 Coefficients DE COllage. - Le coefficient de collage $\alpha$ d'un élément III est fonction de la température du substrat, et des flux incidents. Sa variation en fonction de la température $T_{\mathrm{s}} \mathrm{du}$ substrat pour des valeurs de flux données peut être évaluée quantitativement à partir des variations de la vitesse de croissance du binaire III-V considéré selon $\alpha=v / v_{0}$ où $v_{0}$ est la vitesse maximale correspondant au coefficient de collage égal à 1 .

A titre d'exemple, nous donnons, figure 5 , les variations de la vitesse normalisée $v / v_{0}$ de GaAs en fonction de la température du substrat pendant la croissance de $\mathrm{GaAs}$ et $\mathrm{Al}(x) \mathrm{Ga}(1-x)$ As à diverses concentrations d'aluminium pour des flux d'arsenic et de gallium fixés. Ces résultats montrent que le 
coefficient de collage du gallium est influencé par la présence d'aluminium, en bon accord avec les prédictions thermodynamiques [5].

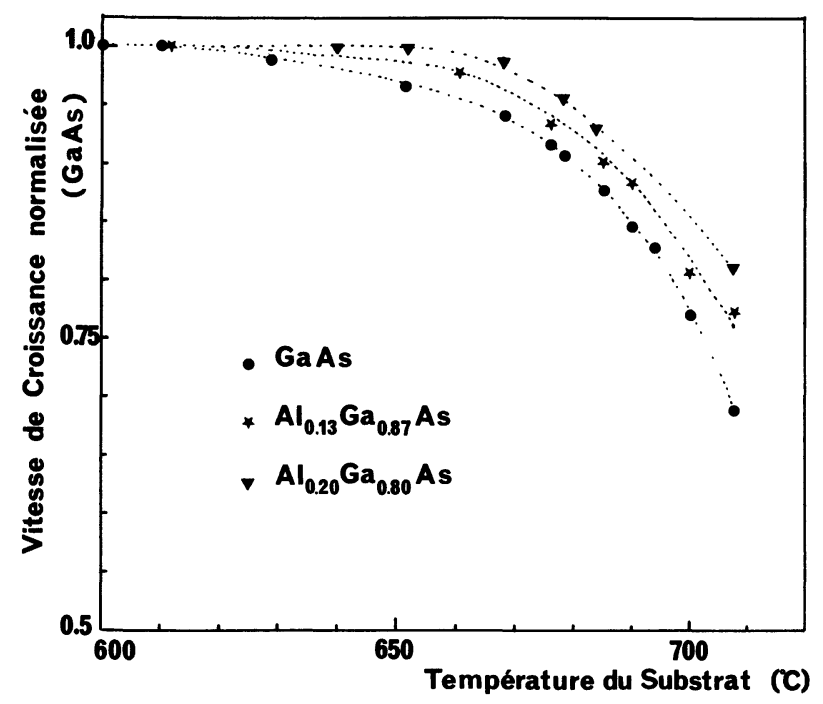

Fig. 5. - Vitesse normalisée de GaAs en fonction de la température de croissance dans le cas de GaAs/GaAs (•), $\mathrm{Al}(0,13) \mathrm{Ga}(0,87) \mathrm{As} / \mathrm{GaAs}(\star)$ et $\mathrm{Al}(0,20) \mathrm{Ga}(0,80) \mathrm{As} /$ GaAs $(\nabla)$, pression dans le flux d'arsenic $1,3 \times$ $10^{-4}$ torr, $v(\mathrm{GaAs})=1,2 \mathrm{MC} / \mathrm{s}$ (compositions d'alliage et vitesse de croissance données à $600^{\circ} \mathrm{C}$ ).

[GaAs normalized growth rate versus growth temperature in the case of $\mathrm{GaAs} / \mathrm{GaAs}(\bullet), \mathrm{Al}(0.13) \mathrm{Ga}(0.87)$ As/GaAs ( $\star$ ) and $\mathrm{Al}(0.20) \mathrm{Ga}(0.80) \mathrm{As} / \mathrm{GaAs}(\boldsymbol{\nabla})$, arsenic beam equivalent pressure $1.3 \times 10^{-4}$ torr, growth rate of $\mathrm{GaAs} 1.2 \mathrm{ML} / \mathrm{s}$, (alloy compositions and growth rate given at $\left.600{ }^{\circ} \mathrm{C}\right)$.]

\section{Informations sur l'interface de croissance.}

5.1 Système (Al, Ga)As/GaAs. - Pour un couple donné $P(\mathrm{As}), T$ (substrat), on peut observer les diagrammes de diffraction de surfaces dites statiques, hors croissance, mais sous flux d'arsenic et après lissage, ou de surfaces dites dynamiques c'est-à-dire en cours de croissance. On définit également les intensités réfléchies $I_{\mathrm{s}}$ et $I_{\mathrm{d}}$ respectivement observées en statique et en dynamique une fois le régime permanent atteint et correspondant à la rugosité «statique » et «dynamique » de la surface. Ces différentes surfaces pourront donc être comparées d'après le diagramme de diffraction observé et l'intensité de la tache spéculaire selon un azimut fixé. Du point de vue de la rugosité de surface, les conditions optimales de croissance correspondront à l'obtention d'un maximum de l'intensité moyenne de la tache spéculaire. Dans le cas d'une croissance réalisée de façon classique sans interruptions, le paramètre important à considérer est l'intensité dynamique $I_{\mathrm{d}}$, alors que pour une croissance avec interruptions et lissages successifs l'intensité statique $I_{\mathrm{s}}$ devient le paramètre essentiel.

5.1.1 Etude des interfaces de croissance GaAs/GaAs pour différentes reconstructions de surface. - Différentes reconstructions de surface ont été observées en statique ou en dynamique pour différentes températures de substrat à pression d'arsenic constante (Fig. 6) ou pour différentes pressions d'arsenic à température de substrat constante (Fig. 7). Ainsi on peut observer successivement les reconstructions $(3 \times 1),(2 \times 4)$ et $c(4 \times 4)$, relatives à un recouvrement croissant en arsenic de la

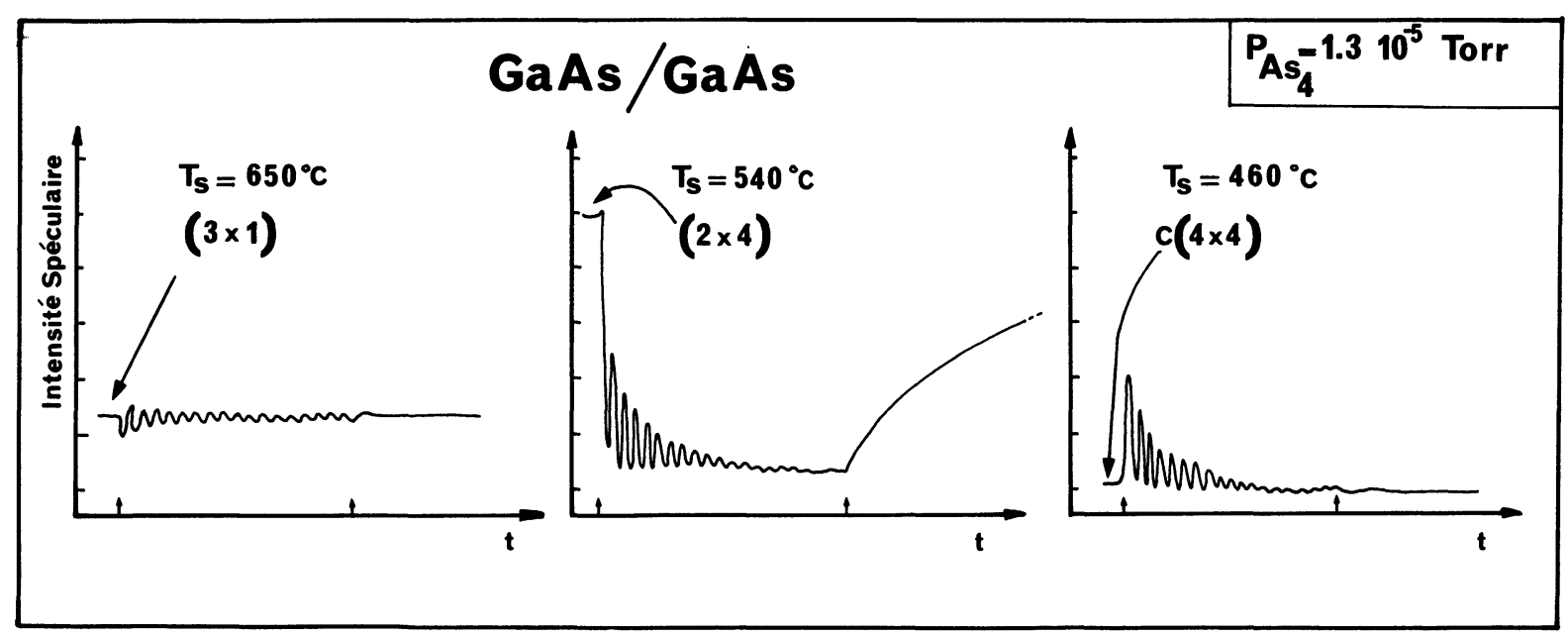

Fig. 6. - Comparaison quantitative des intensités statiques et dynamiques, $I_{\mathrm{s}}$ et $I_{\mathrm{d}}$, des oscillations de croissance de GaAs à différentes températures de substrat (azimut [110], pression dans le flux d'arsenic $1,3 \times 10^{-4}$ torr, $v=1,2 \mathrm{MC} / \mathrm{s}$, les flèches portées en abscisse correspondent au début' et à l'arrêt de la croissance).

[Quantitative comparison of static and dynamic intensities $I_{\mathrm{s}}$ and $I_{\mathrm{d}}$, of GaAs RHEED intensity oscillations at different substrate temperatures ([110] azimuth, arsenic beam equivalent pressure $1.3 \times 10^{-4}$ torr, growth rate $1.2 \mathrm{ML} / \mathrm{s}$, arrows in abscissa correspond to the beginning and the end of the growth).] 


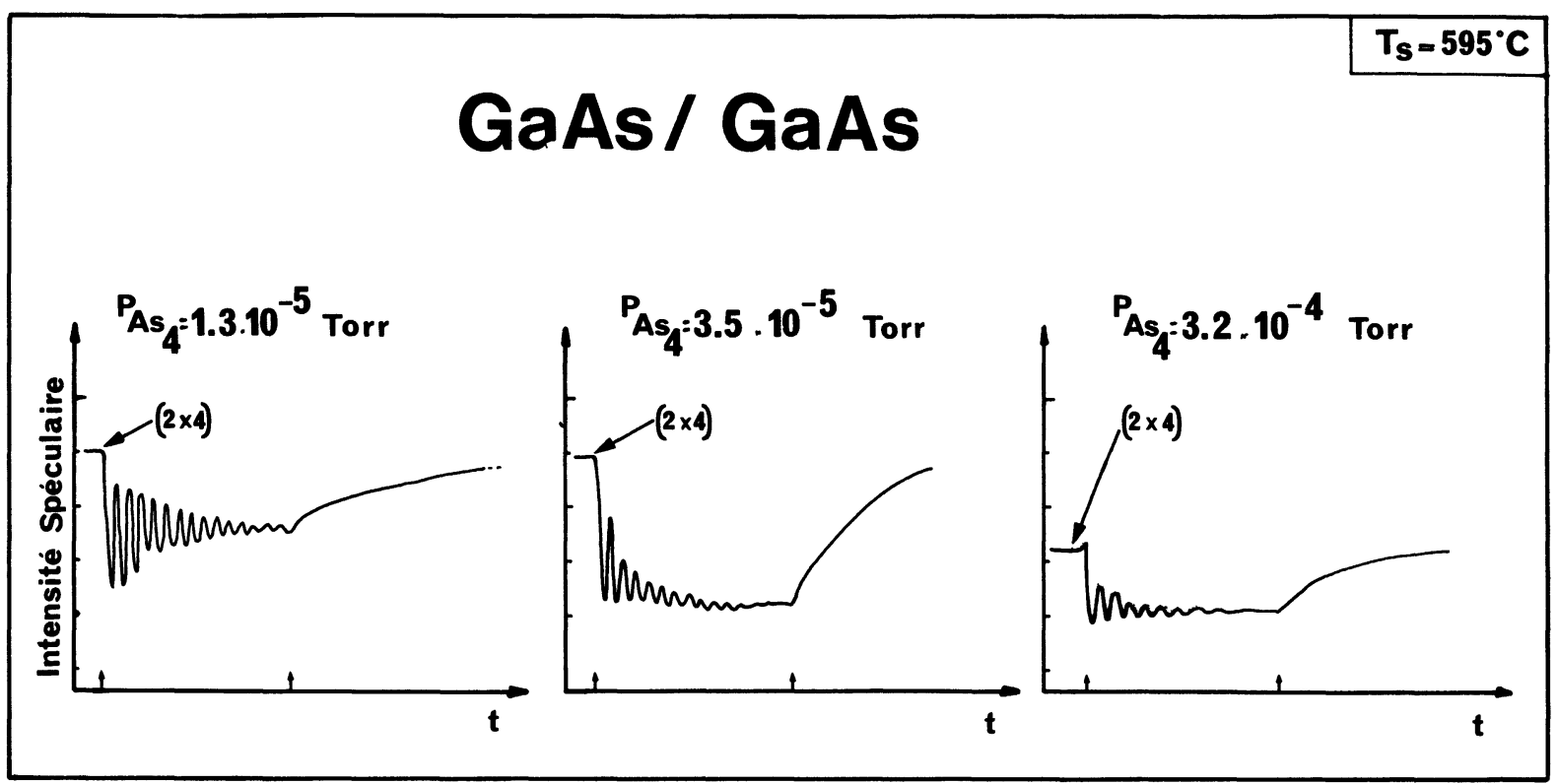

Fig. 7. - Comparaison quantitative des intensités statiques et dynamiques, $I_{\mathrm{s}}$ et $I_{\mathrm{d}}$, des oscillations de croissance de GaAs à différentes pressions dans le flux d'arsenic (azimut [110], $T_{\mathrm{s}}=595^{\circ} \mathrm{C}, v=1,2 \mathrm{MC} / \mathrm{s}$, les flèches portées en abscisse correspondent au début et à l'arrêt de la croissance).

[Quantitative comparison of static and dynamic intensities $I_{\mathrm{s}}$ and $I_{\mathrm{d}}$, of GaAs RHEED intensity oscillations at different arsenic beam equivalent pressures ([110] azimuth, $T_{\mathrm{s}}=595^{\circ} \mathrm{C}$, growth rate $1.2 \mathrm{ML} / \mathrm{s}$, arrows in abscissa are corresponding to the beginning and the end of growth).]

surface, soit en diminuant la température du substrat à pression d'arsenic constante, soit en augmentant la pression d'arsenic à température de substrat constante. L'intensité de la tache spéculaire enregistrée avant la croissance à partir de ces trois reconstructions de la surface de départ, permet de comparer quantitativement l'état de rugosité des surfaces statiques. Au vu de la figure 6, on en déduit une plus forte rugosité pour les surfaces présentant des reconstructions $(3 \times 1)$ ou $c(4 \times 4)$ que pour celles présentant une reconstruction $(2 \times 4)$. Le maximum de réflectivité a été obtenu pour une surface statique présentant une reconstruction $(2 \times 4)$. En prenant pour hypothèse que cette surface de rugosité minimale correspond à une surface quasi stœchiométrique, l'augmentation de rugosité observée pour les surfaces $(3 \times 1)$ et $c(4 \times 4)$ peut être respectivement reliée à un excès de gallium ou d'arsenic à la surface avant croissance.

De plus l'observation du transitoire de croissance, concernant la première demi-oscillation (Fig. 6), fournit les informations suivantes :

- diminution de l'intensité réfléchie pour une surface présentant une reconstruction $(3 \times 1)$ en statique,

- augmentation de l'intensité réfléchie pour une surface présentant une reconstruction $c(4 \times 4)$ en statique (en accord avec les Réf. [6] et [7],

- légère augmentation ou diminution de l'intensité réfléchie pour une surface présentant une recons- truction $(2 \times 4)$ en statique selon l'écart en pression d'arsenic par rapport à l'obtention des surfaces $(3 \times 1)$ et $c(4 \times 4)$ statiques (en accord avec la Réf. [7]).

Dans le cas d'une surface statique $(3 \times 1)$, présentant un défaut d'arsenic, le gallium adsorbé à l'initiation de la croissance augmente encore la proportion relative de $\mathrm{Ga}$ par rapport à As et donc la rugosité, ce qui se traduit par l'existence d'un transitoire décroissant. Au contraire l'excès d'arsenic présent sur une surface $c(4 \times 4)$ est incorporé en GaAs dès l'arrivée des atomes de gallium, ce qui se traduit par l'existence d'un transitoire croissant. La surface $(2 \times 4)$ étant située de façon intermédiaire quant à la population d'arsenic adsorbé, on peut observer l'un ou l'autre des transitoires. L'étude de ces transitoires permet donc d'atteindre la pression d'arsenic optimale pour des conditions de croissance données.

Au-delà du transitoire, la comparaison des intensités réfléchies $I_{\mathrm{s}}$ et $I_{\mathrm{d}}$ fournit des indications sur l'évolution de la rugosité de la surface entre la phase statique et la phase dynamique une fois le régime permanent de croissance atteint (il a été vérifié que la luminosité induite par l'ouverture de la cellule de gallium ne perturbe pas de manière significative la valeur mesurée de $I_{\mathrm{d}}$ ). Cette comparaison n'est toutefois valable que si les effets de diffraction dynamique restent faibles, ce qui a été remis en question récemment [2]. Lorsque la surface statique 
est reconstruite $(3 \times 1)$, l'intensité statique $I_{\mathrm{s}}$ est égale à l'intensité dynamique $I_{\mathrm{d}}$. On peut donc considérer que la rugosité demeure constante de la phase statique à la phase dynamique. Par contre pour une surface statique $(2 \times 4)$, on observe en croissance que $I_{\mathrm{d}}$ est inférieur à $I_{\mathrm{s}}$. La phase dynamique est plus rugueuse que la phase statique après lissage suite à l'instauration d'un front de croissance conduisant à une augmentation de la densité de marches, de telle sorte que $I_{\mathrm{d}}<I_{\mathrm{s}}$. Dans le cas d'une surface statique $c(4 \times 4), I_{\mathrm{d}}$ est supérieur ou égal à $I_{\mathrm{s}}$. Cette reconstruction correspond en effet à une surface statique plus rugueuse, des molécules d'arsenic étant adsorbées à la surface provoquant un accroissement de la diffusion, qui a été interprété comme la conséquence de la formation de marches supplémentaires [6].

Enfin, il est possible de comparer quantitativement les valeurs de $I_{\mathrm{d}}$. On peut observer à la figure 6 que la croissance de GaAs présente une intensité $I_{\mathrm{d}}$ maximale dans le cas d'une reconstruction de surface $(3 \times 1)$, la valeur de $I_{\mathrm{d}}$ diminuant au fur et à mesure que l'on diminue la température de croissance ; d'autre part, on remarque également que la valeur de $I_{\mathrm{d}}$ diminue lorsque l'on augmente la pression d'arsenic (Fig. 7). Dans les deux cas, diminuer la température de croissance ou augmenter la pression d'arsenic revient à limiter la diffusion du gallium en surface et donc à augmenter la rugosité de la surface [7].

5.1.2 Comparaison des interfaces de croissance $\mathrm{GaAs} / \mathrm{GaAs}$ et $\mathrm{Al}(x) \mathrm{Ga}(1-x) \mathrm{As} / \mathrm{GaAs}$. - Une étude comparative des comportements des interfaces $\mathrm{GaAs} / \mathrm{GaAs}$ et $\mathrm{Al}(x) \mathrm{Ga}(1-x) \mathrm{As} / \mathrm{GaAs}$ à différentes températures a également été entreprise. Comme on peut le voir sur la figure 8 , la valeur de l'intensité moyenne demeure constante dans le cas de croissances de GaAs/GaAs. En ce qui concerne $\mathrm{Al}(x) \mathrm{Ga}(1-x)$ As cette valeur moyenne décroît brutalement pour des croissances ayant lieu à des températures comprises entre 630 et $670^{\circ} \mathrm{C}$ (Fig. 8). Cet intervalle de température correspond à la zone de température de croissance dite "interdite " [810], pour laquelle on observe l'apparition d'une rugosité de surface attribuée récemment à une ségrégation du gallium en surface [11-13].

5.2 SYSTÈME (Al, Ga)InAs/InP. - Le phénomène des oscillations de RHEED a également été observé dans le cadre de la croissance de ce système sur InP (Fig. 9). La croissance des ternaires $\mathrm{Al}(0,48)$ $\operatorname{In}(0,52)$ As et $\mathrm{Ga}(0,47) \operatorname{In}(0,53)$ As en accord de maille sur InP nécessite une bonne connaissance des flux d'éléments III, en effet une variation de $1 \%$ de la composition des ternaires entraîne un désaccord paramétrique relatif entre la couche et le substrat de $7 \times 10^{-4}$. Cependant la connaissance des flux émis n'est pas suffisante notamment pour la croisance de

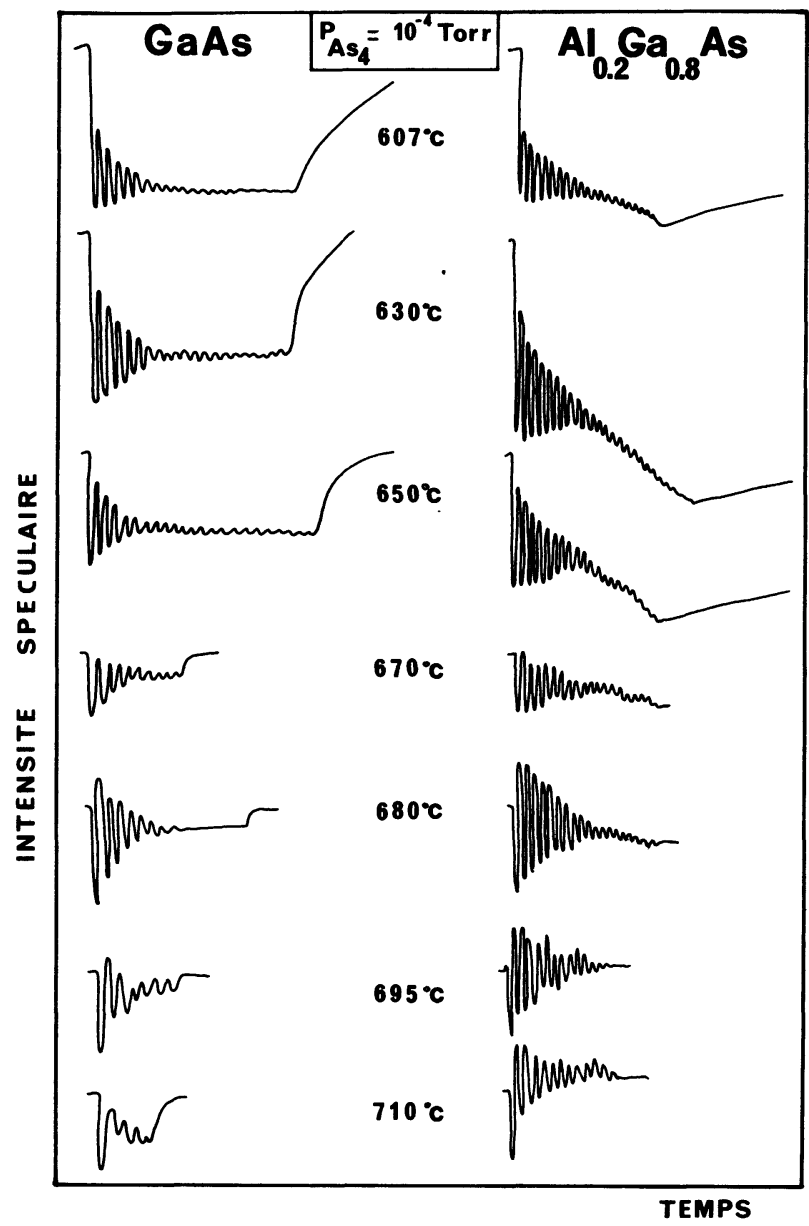

Fig. 8. - Etude comparative des oscillations de croissance de $\mathrm{GaAs} / \mathrm{GaAs}$ et de $\mathrm{Al}(0,20) \mathrm{Ga}(0,80) \mathrm{As} / \mathrm{GaAs}$ (composition d'alliage donnée à $600^{\circ} \mathrm{C}$ ), à différentes températures de substrat (azimut [110] pression dans le flux d'arsenic $1,3 \times 10^{-4}$ torr).

[Comparative study of RHEED intensity oscillations of $\mathrm{GaAs} / \mathrm{GaAs}$ and $\mathrm{Al}(0.20) \mathrm{Ga}(0.80) \mathrm{As} / \mathrm{GaAs}$ (alloy composition given at $600^{\circ} \mathrm{C}$ ), at different substrates temperatures ([110] azimuth, arsenic beam equivalent pressure $1.3 \times 10^{-4}$ torr).]

$\mathrm{Al}(1-x) \operatorname{In}(x) \mathrm{As} / \mathrm{InP}$ qui est généralement effectuée dans une zone de température où le coefficient de collage de l'indium n'est plus égal à 1.

\subsubsection{Détermination de la composition d'alliage.}

- Les cellules d'aluminium et de gallium étant étalonnées comme indiqué préalablement (voir 4.2) et les croissances de $\mathrm{Al}(1-x) \operatorname{In}(x) \mathrm{As}$ et de $\mathrm{Ga}(1-x) \operatorname{In}(x)$ As étant réalisées à des températures inférieures à $600{ }^{\circ} \mathrm{C}$ pour lesquelles les coefficients de collage de $\mathrm{Ga}$ et $\mathrm{Al}$ sont égaux à 1 , la connaissance de la vitesse de croissance du ternaire est suffisante pour la détermination de la fraction molaire de InAs et du coefficient de collage de l'indium. Sa variation en fonction de la température du substrat a été étudiée au moyen des oscillations de RHEED pendant la croissance de $\mathrm{Al}(1-x) \operatorname{In}(x) \mathrm{As} / \mathrm{InP}$ et de $\mathrm{Al}(1-x) \operatorname{In}(x)$ As / 


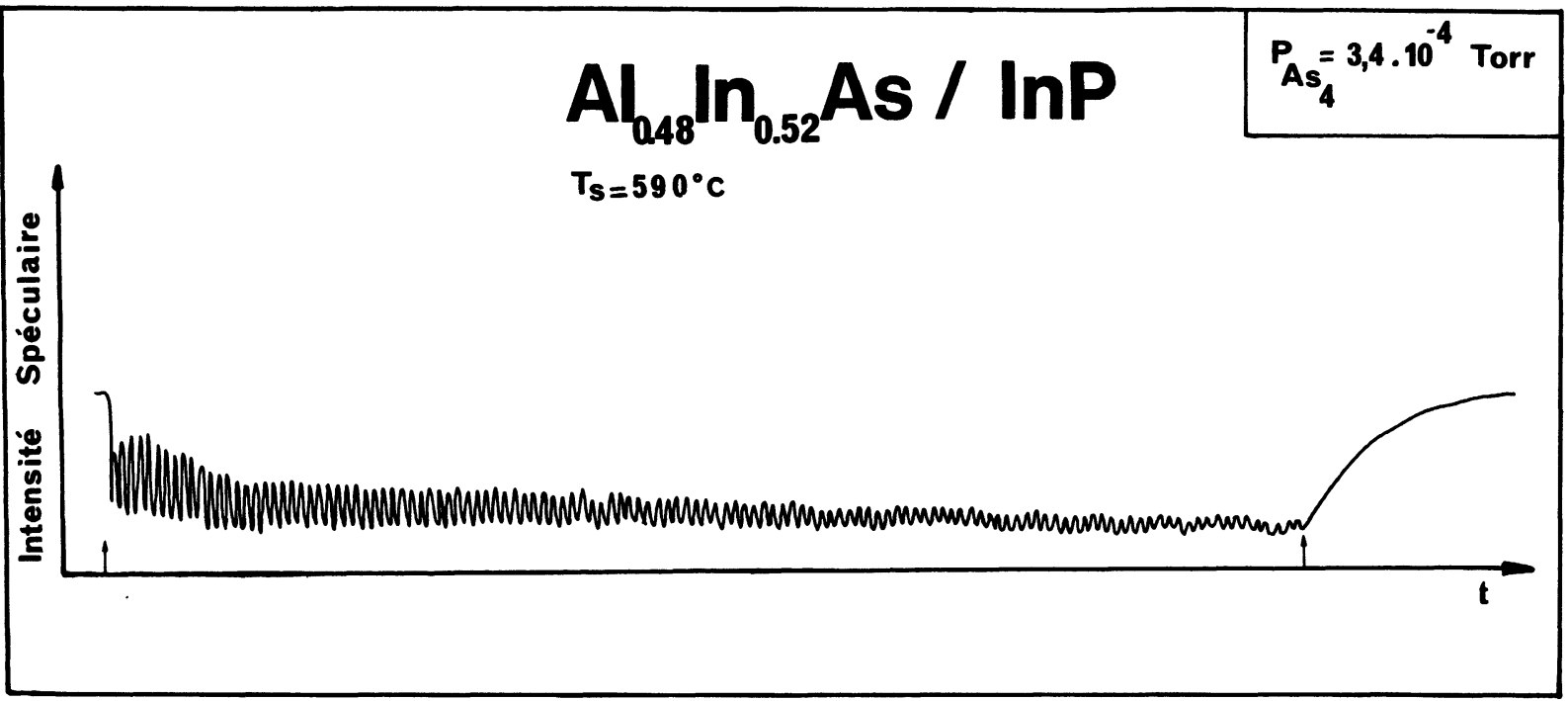

Fig. 9. - Oscillations de RHEED pendant la croissance de $\mathrm{Al}(0,48) \operatorname{In}(0,52) \mathrm{As} / \mathrm{InP}$ et récupération de l'intensité initiale à l'arrêt de croissance (azimut [110], $T_{\mathrm{s}}=590^{\circ} \mathrm{C}$, pression dans le flux d'arsenic $3,5 \times 10^{-4}$ torr, $v=$ $0,70 \mathrm{MC} / \mathrm{s}$, les flèches portées en abscisse correspondent au début et à l'arrêt de la croissance).

[RHEED oscillations during $\mathrm{Al}(0.48) \operatorname{In}(0.52) \mathrm{As} / \mathrm{InP}$ growth and recovering of the initial intensity at the end of the growth ([110] azimuth, $T_{\mathrm{s}}=590^{\circ} \mathrm{C}$, arsenic beam equivalent pressure $3.5 \times 10^{-4}$ torr, growth rate $0.70 \mathrm{ML} / \mathrm{s}$ arrows in abscissa correspond to the beginning and the end of the growth).]

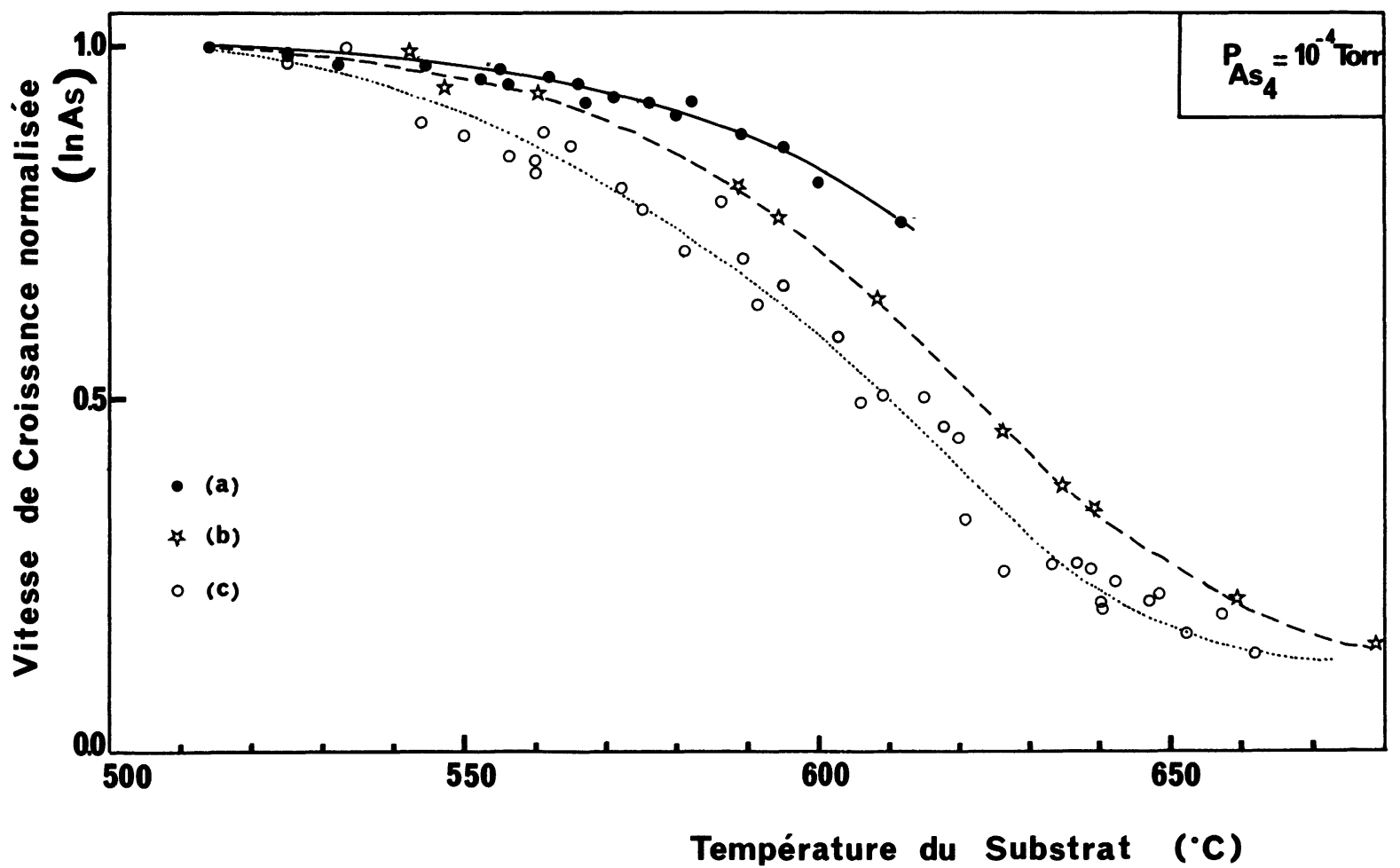

Fig. 10. - Vitesse normalisée de InAs en fonction de la température de croissance dans le cas de $\mathrm{Al}(0,48) \operatorname{In}(0,52)$ As $/ \mathrm{InP}(\mathrm{a})$ et de $\mathrm{Al}(0,70) \operatorname{In}(0,30) \mathrm{As} / \mathrm{GaAs}(\mathrm{b}, \mathrm{c}), v_{0}(\mathrm{InAs})=0,34 \mathrm{MC} / \mathrm{s}$ pour a et $\mathrm{b}$ et $0,17 \mathrm{MC} / \mathrm{s}$ pour $\mathrm{c}$, compositions d'alliage et vitesses de croissance données à $500^{\circ} \mathrm{C}$, pression dans le flux d'arsenic $1,0 \times 10^{-4}$ torr.

[InAs normalized growth rate versus growth temperature in the case of $\mathrm{Al}(0.48) \operatorname{In}(0.52) \mathrm{As} / \mathrm{InP}(\mathrm{a})$ and of $\mathrm{Al}(0.70) \operatorname{In}(0.30)$ As $/ \mathrm{GaAs}(\mathrm{b}, \mathrm{c})$, growth rate of InAs $0.34 \mathrm{ML} / \mathrm{s}$ for $\mathrm{a}$ and $\mathrm{b}$ and $0.17 \mathrm{ML} / \mathrm{s}$ for $\mathrm{c}$, alloy compositions and growth rates given at $500^{\circ} \mathrm{C}$, arsenic beam equivalent pressure $1.0 \times 10^{-4}$ torr.] 
GaAs. Les résultats, obtenus pour une pression dans le flux d'arsenic constante $\left(1 \times 10^{-4}\right.$ torr $)$, sont reportés à la figure 10 . Dans le cas de la croissance sur substrat InP (Fig. 10a), la composition de l'alliage correspond à l'accord paramétrique couche substrat $(x=0,48)$ pour la température de croissance standard de $580^{\circ} \mathrm{C}$. La composition de l'alliage épitaxié sur GaAs est $x=0,3$ pour $T_{\mathrm{s}} \leqslant 500^{\circ} \mathrm{C}$ (Fig. 10b, c). Cette composition équivaut à un désaccord paramétrique de l'ordre de $2,3 \times 10^{-2}$. Les figures $10 \mathrm{a}$ et $\mathrm{b}$ correspondent à un même flux d'indium (pression équivalente dans le flux 2,6 $\times$ $10^{-7}$ torr), alors que ce flux est divisé par 2 pour la figure 10c (pression équivalente dans le flux $1,3 \times$ $10^{-7}$ torr et donc vitesse $v_{0}$ (InAs) deux fois plus petite). La diminution du flux d'indium incident provoque l'accroissement de l'influence de la désorption d'indium et explique simplement la différence observée entre les points expérimentaux $b$ et $c$, en accord avec l'analyse thermodynamique en termes d'équilibre chimique. Pour comparer les expériences concernant AlInAs/InP (Fig. 10a) et AlInAs/GaAs (Fig. 10b), réalisés à flux incident d'indium constant, il faut tenir compte de deux effets : l'un dû à la différence de la fraction molaire AlAs dans les alliages considérés, l'autre dû au caractère contraint de l'alliage épitaxié sur GaAs. Par analogie avec l'influence de l'aluminium sur le coefficient de collage de $\mathrm{Ga}$ dans $\mathrm{Al}(x) \mathrm{Ga}(1-x) \mathrm{As}$, on peut considérer que la plus forte proportion d'aluminium dans le cas de l'alliage épitaxié sur GaAs $\left(x_{\mathrm{Al}}=\right.$ 0,7 contre $x_{\mathrm{Al}} \simeq 0,5$ sur InP), limite l'effet de la désorption de l'indium et ne va donc pas dans le sens de la différence observée entre les points a et $b$. Il semble donc que le désaccord paramétrique augmente les effets de désorption, l'énergie de contrainte défavorisant la formation de liaisons InAs (essentiellement responsable de l'écart de paramètre) par rapport à $\mathrm{Al}$-As.

5.2.2 Influence de la température de croissance. - Les croissances de $\mathrm{Ga}(1-x) \operatorname{In}(x)$ As et $\mathrm{Al}(1-x) \operatorname{In}(x)$ As sur substrat InP ont été effectuées à des températures allant de 485 à $570{ }^{\circ} \mathrm{C}$ pour une pression d'As fixée à $1 \times 10^{-4}$ torr (Fig. 11). Un parallèle a pu être établi entre les oscillations enregistrées sur $\mathrm{Al}(1-x) \operatorname{In}(x) \mathrm{As} / \mathrm{InP}$ et $\mathrm{Ga}(1-x) \operatorname{In}(x)$ As/InP d'une part et GaAs/GaAs d'autre part. A $485^{\circ} \mathrm{C}$, les oscillations de RHEED enregistrées pour les deux matériaux (surface statique reconstruite $(4 \times 3))$ sont similaires aux oscillations de GaAs dans le cas d'un excès d'arsenic en surface (surface statique reconstruite $c(4 \times 4)$. Pour les températures supérieures, on observe des oscillations typiques d'une reconstruction $(2 \times 4)$ sur $\mathrm{GaAs}$, cette reconstruction étant également observée sur $\mathrm{Al}(1-x) \operatorname{In}(x)$ As et $\mathrm{Ga}(1-x) \operatorname{In}(x)$ As. A $565^{\circ} \mathrm{C}$, la croissance de $\mathrm{Ga}(1-x) \operatorname{In}(x) \mathrm{As} / \mathrm{InP}$ pré-

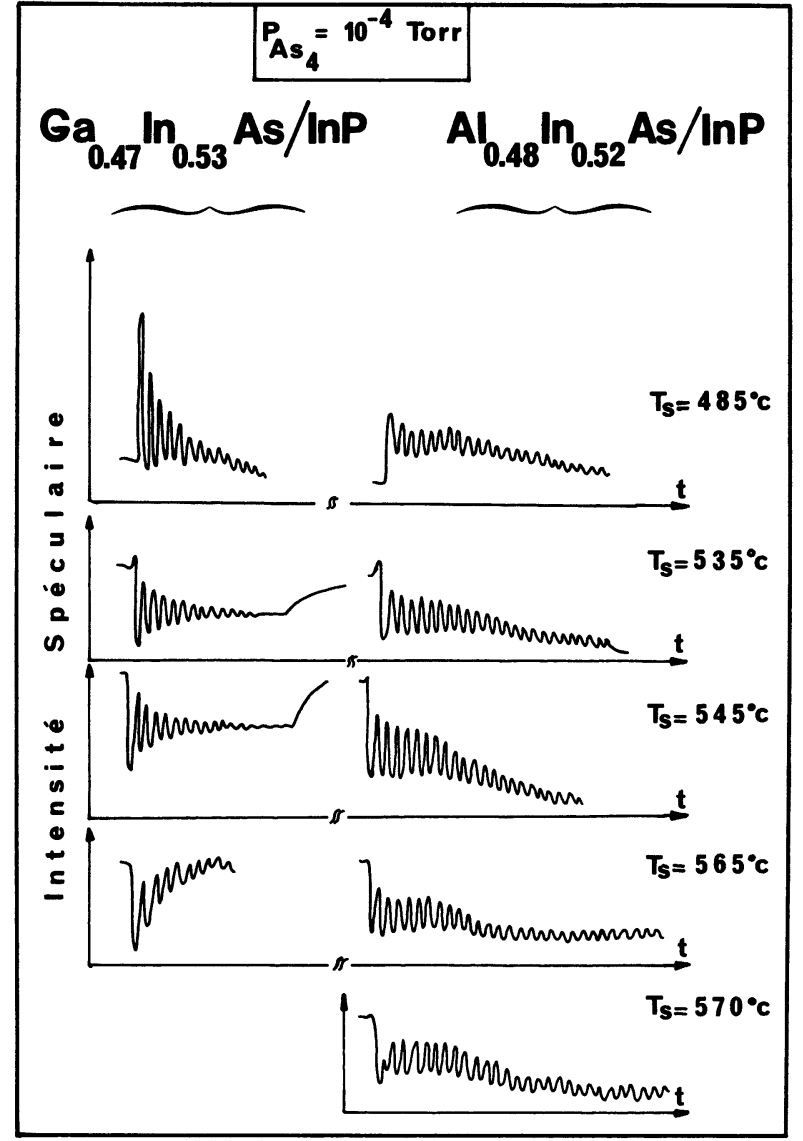

Fig. 11. - Influence de la température de croissance sur les oscillations de RHEED enregistrées pendant les croissances de $\mathrm{Ga}(0,47) \operatorname{In}(0,53) \mathrm{As} / \mathrm{InP}$ et $\mathrm{Al}(0,48) \operatorname{In}(0,52)$ As/InP (azimut [110], pression dans le flux d'arsenic $1 \times 10^{-4}$ torr, $v=0,70 \mathrm{MC} / \mathrm{s}$.

[Influence of the growth temperature on RHEED oscillations recorded during $\mathrm{Ga}(0.47) \operatorname{In}(0.53) \mathrm{As} / \mathrm{InP}$ and $\mathrm{Al}(0.48) \mathrm{In}(0.52) \mathrm{As} / \mathrm{InP}$ growths, ([110] azimuth, equivalent beam pressure of arsenic $1 \times 10^{-4}$ torr, growth rate $0.70 \mathrm{ML} / \mathrm{s})$.]

sente des oscillations typiques d'une quantité d'arsenic disponible à la surface juste suffisante. On peut noter qu'à cette même température $\operatorname{Al}(1-x)$ $\operatorname{In}(x) \mathrm{As} / \mathrm{InP}$ ne présente pas un spectre d'oscillations similaire, en effet les alliages à base d'aluminium peuvent être réalisés avec un flux d'arsenic moindre, cette différence a été également observée entre les croissances de GaAs/GaAs. et de $\mathrm{Al}(x) \mathrm{Ga}(1-x) \mathrm{As} / \mathrm{GaAs}$. Au-delà de $565^{\circ} \mathrm{C}$ il est difficile d'observer des oscillations de RHEED pour GaInAs/InP alors qu'elles peuvent l'être jusqu'à $615^{\circ} \mathrm{C}$ pour AlInAs/InP, ceci est probablement dû à la différence de mobilité des atomes de $\mathrm{Ga}$, In et $\mathrm{Al}$ à la surface.

6. Informations tirées de la forme détaillée des oscillations.

6.1 SymÉtRie Des oscillations. - On observe dans certains cas une dissymétrie des oscillations : le 


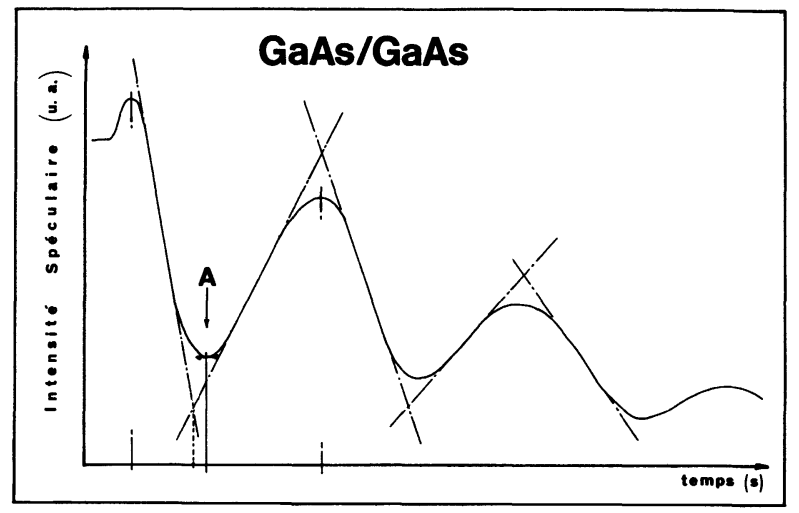

Fig. 12. - Exemple de dissymétrie des oscillations enregistrées pendant la croissance de $\mathrm{GaAs} / \mathrm{GaAs}$ (azimut $\left.[100], T_{\mathrm{s}}=550^{\circ} \mathrm{C}, v(\mathrm{GaAs})=0,1 \mathrm{MC} / \mathrm{s}\right)$.

[Example of dissymmetric oscillations recorded during GaAs $/ \mathrm{GaAs}$ growth ([110] azimuth, $T_{\mathrm{s}}=550^{\circ} \mathrm{C}$, growth rate $0.1 \mathrm{ML} / \mathrm{s})$.]

minimum de réflexion (point $\mathrm{A}$, Fig. 12) ne correspond plus à une demi-oscillation. Il est possible que cette dissymétrie provienne en partie des phénomènes d'interaction des électrons rasants avec la surface. En effet, dès les premiers stades de la croissance par nucléation 2D, des éléments 'diffuseurs, sous la forme d'atomes, de molécules ou de cristallites 2D sont présents à la surface. Il existe alors une zone d'ombre dans la direction du faisceau d'électrons, associée à la taille de ces îlots. L'intensité réfléchie n'est plus sensible aux éléments diffusants arrivant dans cette zone d'ombre, ceci induit une rugosité apparente (inférieure ou égale à la rugosité réelle). Cet effet pourrait expliquer le décalage observé du minimum de l'intensité réfléchie par rapport à la demi-période [14].

Cependant, s'il semble bien que ce phénomène crée une dissymétrie dans la forme des oscillations, il n'en est sans doute pas le seul responsable. En effet l'amortissement général des oscillations souvent dissymétrique d'une part, et les structures que l'on observe dans la forme même des oscillations d'autre part, doivent y contribuer.

6.2 StRuCtures OBSERVÉES DANS LES OSCILlATIONS. - Lorsqu'il est possible de détailler les enregistrements des oscillations, on observe de façon reproductible (Fig. 13a et b) des structures encore inexpliquées. Un travail est actuellement en cours sur la possibilité de traiter ces oscillations par transformée de Fourier.

\section{Conclusion.}

Les oscillations de RHEED sont un outil puissant de caractérisation de l'interface de croissance et relati-

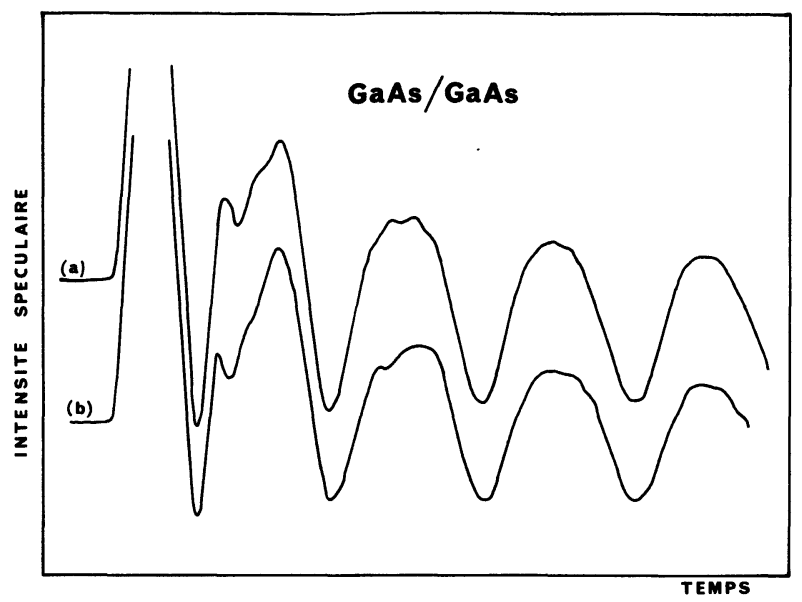

Fig. 13. - Structures observées dans les oscillations enregistrées pendant la croissance de $\mathrm{GaAs} / \mathrm{GaAs}$ (azimut [110], $\left.T_{\mathrm{s}}=550^{\circ} \mathrm{C}, v(\mathrm{GaAs})=1,5 \mathrm{MC} / \mathrm{s}\right)$ pour deux expériences consécutives a et $b$.

[Structures observed in RHEED oscillations recorded during $\mathrm{GaAs} / \mathrm{GaAs}$ growth ([110] azimuth, $T_{\mathrm{s}}=550^{\circ} \mathrm{C}$, growth rate $1.5 \mathrm{ML} / \mathrm{s}$ ) for two consecutive experiments (a and b).]

vement simple d'emploi. Outre les mesures pratiquement instantanées de la vitesse de croissance, de la composition d'alliage et des coefficients de collage des éléments III, il paraît possible en utilisant cette technique d'optimiser les conditions de croissance et notamment la température du substrat, la pression d'arsenic et la vitesse de croissance, en étudiant les valeurs absolues de l'intensité réfléchie en statique et en dynamique, ainsi que le transitoire au départ de la croissance. Cependant, la caractérisation effectuée par ces oscillations est essentiellement de type morphologique, il est nécessaire de vérifier les qualités électriques et optiques de ces matériaux élaborés dans les conditions dites optimisées.

L'étude des oscillations de RHEED, apportant une meilleure connaissance de l'interface de croissance, devrait permettre de progresser rapidement dans la compréhension des mécanismes de la croissance. De plus, l'utilisation de cette technique de contrôle est tout à fait adaptée à la réalisation d'interfaces abruptes et donc de super-réseaux ou de structures à multiples puits quantiques.

\section{Remerciements.}

Ce travail a été partiellement soutenu par la Compagnie Générale d'Electricité dans le cadre du contrat $n^{\circ}$ 305/C/85 des Laboratoires de Marcoussis. Les auteurs remercient A. Barski (ISA Riber), P. Claverie (CNRS-Lure-Orsay), A. Leycuras, F. Raymond et A. Salètes pour de nombreuses discussions et O. Tottereau pour son efficace aide technique. 


\section{Bibliographie}

[1] Neave, J. H., Joyce, B. A., Dobson, P. J., Norton, N., Appl. Phys. A 31 (1983) 1.

[2] Dobson, P. J., Joyce, B. A., Neave, J. H., ZHANG, J., J. Cryst. Growth 81 (1987) 1.

[3] Madhukar, A., Chen, P., Voillot, F., Thomsen, M., KIM, J. Y., TANG, W. C., Ghaisas, S. V., J. Cryst. Growth 81 (1987) 26.

[4] Neave, J. H., Joyce, B. A., Dobson, P. J., Appl. Phys. A 34 (1984) 179.

[5] Heckingbottom, R., J. Vac. Sci. Technol. B (1985) 572.

[6] Briones, F., Golmayo, D., Gonzalez, L., DE Miguel, J. L., Jpn. J. Appl. Phys. 24, 6 (1985) 1478.

[7] Charasse, M. N., Thèse Université P. et M. Curie (Paris 1985).

[8] Wang, W. I., Judaprawira, S., Wood, C. E. C., Eastman, L. F., Appl. Phys. Lett. 38 (1981) 708.
[9] Morkoc, H., Drummond, T. J., KopP, W., Fisher, R., J. Electrochem. Soc. 129 (1982) 824.

[10] Alexandre, F., Goldstein, L., Leroux, G., JonCOUR, M. C., Thibierge, H., RAO, E. V. K., J. Vac. Sci. Technol. B 3 (1985) 950.

[11] Stall, R. A., Zilko, J., Swaminathan, V., SchuMAKER, N., J. Vac. Sci. Technol. B 3 (1985) 524.

[12] Massies, J., Rochette, J. F., Delescluse, P., J. Vac. Sci. Technol. B 3 (1985) 613.

[13] Massies, J., Turco, F., Saletes, A., Contour, J. P., présenté à la $4^{\mathrm{e}}$ Conférence Internationale sur l'Epitaxie par Jets Moléculaires (York, Angleterre, Septembre 1986), J. Cryst. Growth 80 (1987) 307.

[14] Turco, F., Massies, J., Leycuras, A., Contour, J. P., RAYmond, F., Barski, A., et Claverie, P., Comptes rendus du $4^{\mathrm{e}}$ Séminaire d'Epitaxie par Jets Moléculaires Saint-Aygulf, juin 1986 et Leycuras, A., Turco, F., Massies, J., résultats non publiés. 